\title{
Ineffectiveness of visual distinctiveness in enhancing immediate recall
}

\author{
JOAN MCDOWD and STEPHEN MADIGAN \\ University of Southern California, Los Angeles, California
}

\begin{abstract}
Glenberg (1984) and others have theorized that greater recency effects are obtained with auditory as opposed to visual presentation because of greater temporal distinctiveness of items in auditory sequences. We tested a number of ways of enhancing visual distinctiveness, including the use of color, spatial location, and minimized visual interference. None of the seven experiments provided any evidence of improved recall from enhanced visual distinctiveness. In particular, no increase in recency effects was obtained with increased distinctiveness. Additional analyses of pairwise dependency in recall across serial positions also failed to show any evidence of the near-independence of recall of the terminal item that characterizes recall of auditory sequences. Visual-perceptual distinctiveness does not get mapped in any simple way onto memorial distinctiveness in an immediate-serial-recall task.
\end{abstract}

The series of experiments reported in this paper were attempts to influence immediate recall of short lists of visually presented digits and words by experimental operations designed to increase the distinctiveness of some or all of the list items. The research was prompted by recent experimental and theoretical developments that suggest that modality effects in immediate recall may be best understood in terms of differences in the distinctiveness of auditorily and visually presented materials (Glenberg, 1987; Glenberg \& Swanson, 1986). According to this view, the elements of a sequence of auditorily presented events have greater temporal discriminability, or distinctiveness, than a sequence of visually presented events. Differences in recall between modalities is thought to result from more sharply defined memory search sets in the case of auditory presentation.

This approach is a move away from the concept of precategorical acoustic storage (PAS) (Crowder \& Morton, 1969 ) as the most satisfactory account of modality differences, and particularly of the greater recency effect obtained with auditory as opposed to visual presentation. A number of experimental findings have raised serious questions about the adequacy of the PAS model, such as the occurrence of recency effects for mouthed but not pronounced stimuli (Crowder, 1986), and long-term modality effects in which auditory superiority is obtained at intervals much greater than the time periods usually thought to characterize the persistence of PAS or "echoic memory"' (Glenberg, 1984).

Hypothesizing distinctiveness as the locus of modality effects leads to two ways of testing the distinctiveness concept. In the first, one attempts to give visual stimuli the

Correspondence may be addressed to Joan McDowd, Department of Psychology, SGM-501, University of Southern California, Los Angeles, CA 90089-1061. temporally distributed character of auditory stimuli, in one case by having each visual stimulus "unfold" over time (Crowder, 1986). The present experiments were based on a second general approach: Rather than trying to make a visual stimulus more like an auditory stimulus, we attempted to increase visual distinctiveness using stimulus characteristics inherent to the visual modality. These characteristics included color, spatial location, and conditions of minimized visual competition and interference.

\section{EXPERIMENT 1}

The first experiment employed a color-change procedure as a way of increasing the distinctiveness and possibly the recall of the last item in visually presented lists.

\section{Method}

The experiment was based on immediate serial recall of lists of nine digits under two conditions: the standard, or control, condition and the experimental condition, in which the terminal list item was perceptually distinctive.

All testing was preceded by three practice trials under the standard condition. ${ }^{1} \mathrm{~A}$ block of 10 trials in each of the experimental and control conditions was then presented. The order of testing was counterbalanced across subjects. Each block was preceded by a set of instructions specific to that block.

Testing was performed with a microcomputer system that prepared nine-digit sequences (formed by sampling without replacement) independently for each subject, presented instructions and test trials, and gathered and scored responses. Each trial began with a warning signal, followed by presentation of the digit sequence at a rate of one item per second, followed by a test trial in which subjects responded by typing digits on the keyboard. Subjects were required to recall in a forward serial order and to make nine responses per test, guessing if necessary.

The distinctiveness operation in the first study consisted of presenting the last digit in the list in color. In control lists and in the first eight serial positions of experimental lists, the digits were presented as white characters on a black background. In experimental lists, the final (ninth) digit was red on a black background. Subjects were 
informed in the initial instructions that the color change merely signaled the end of the list; no suggestion was made that it was a special item, to be emphasized in rehearsal or recall.

Subjects were 20 college students from an introductory psychology course, who participated for extra course credit. All subjects in subsequent studies were from this same pool. Assignment to normal-distinctive and distinctive-normal testing orders was random.

\section{Results}

The results of Experiment 1 appear in Figure 1 in the form of the serial-position effect on percent correct recall (correct digit in correct serial position). These data have been collapsed over the two test orders (normal-distinct and distinct-normal); in this and later experiments there were positive practice effects for the second-tested condition, but no treatment-order interactions.

It is clear from these data that the color change for the last item in experimental lists had no effect on recall, either of the terminal serial position or of earlier serial positions. Statistically, this took the form of a main effect of serial position $\left[F(8,152)=28.92, M S_{\mathrm{e}}=4.01, p<.001\right]$, but no treatment $(F<1.0)$ or treatment $\times$ serial-position effects $\left[F(8,152)=1.71, M S_{\mathrm{e}}=1.48\right.$, n.s. $]$ in a treatment $X$ serial-position analysis of variance. Putting it in other terms, we failed to obtain a Von Restorff-like effect for the perceptually distinct item, in which such an item is recalled well at the expense of items in other serial positions.

\section{EXPERIMENT 2}

Experiment 2 was an attempt to produce a stronger implementation of the distinctiveness operation. Instead of

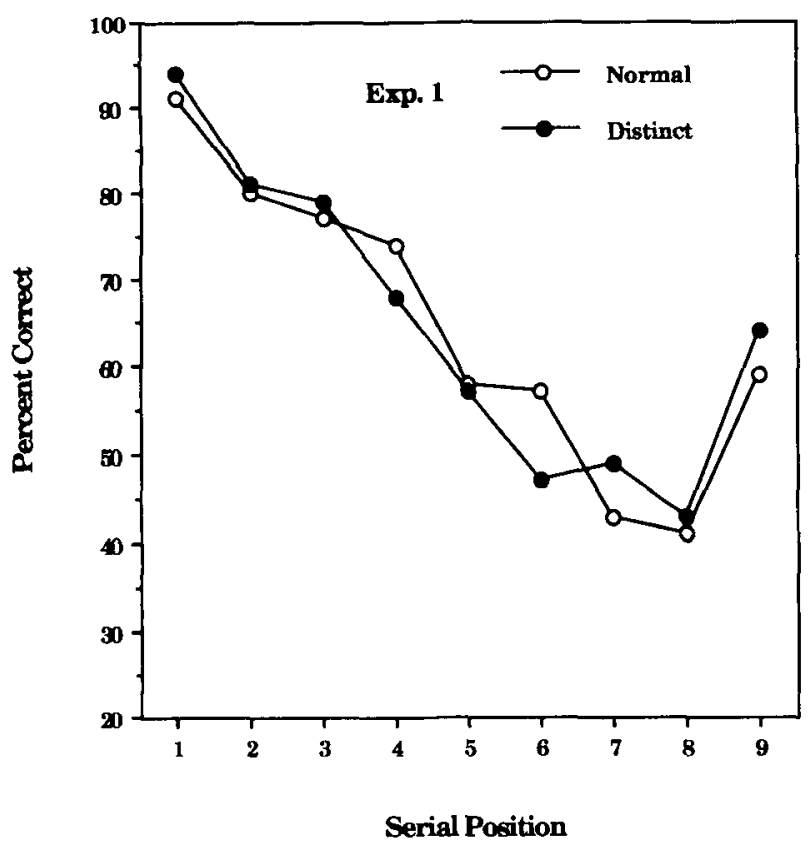

Figure 1. Percent correct ordered recall by serial position for normal and distinct final-item conditions (Experiment 1).

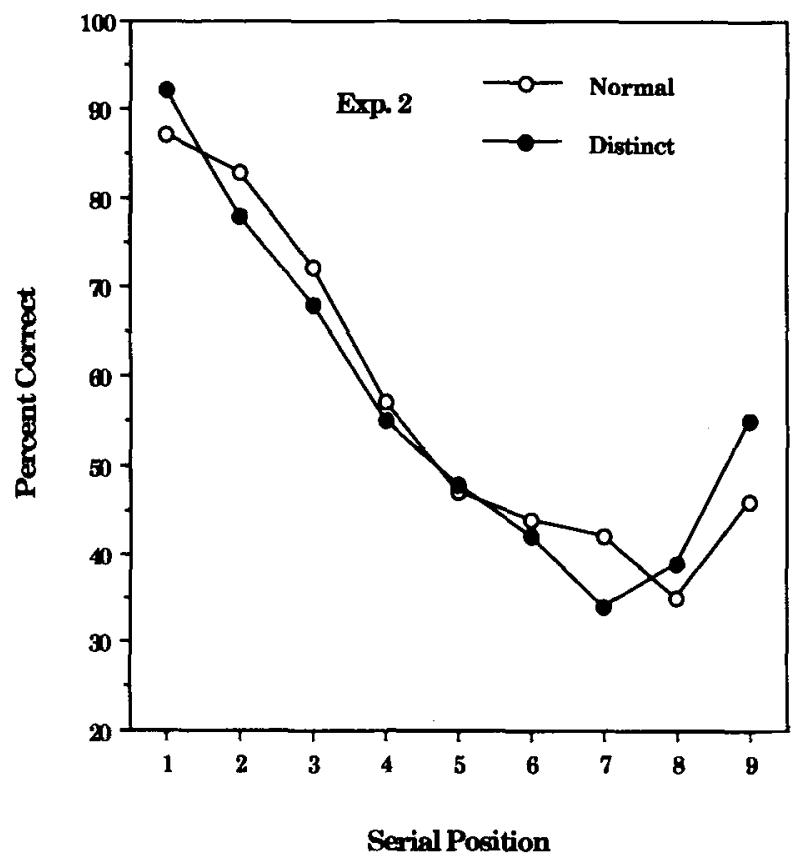

Figure 2. Percent correct ordered recall by serial position for normal and distinct final-screen conditions (Experiment 2).

a color change in a relatively small part of visual field (the final digit in a list), the entire viewing screen changed color on presentation of the last digit.

\section{Method}

The details of procedure were exactly the same as in Experiment 1 , with the exception that presentation of the final list item in the experimental condition was accompanied by a change in the color of the entire screen background (from black to light blue) with no change in the color of the digit.

Subjects were 24 college students, assigned at random to normal-distinct and distinct-normal testing orders.

\section{Results}

Figure 2 presents the serial position effect for normal and distinctive lists. As in Experiment 1, it is clear from these data that the viewing-screen-color change for the list item in experimental lists had no effect on recall. Once again, the only reliable source of variation in the data was serial position $[F(8,184)=54.37, p<.001]$. Neither the effect of treatment $(F<1.0)$ nor the treatment $\times$ serial-position interaction $\left[F(8,184)=1.66, M S_{e}=2.11\right.$, n.s.] reached statistical significance. A test of the simple main effect at the ninth serial position was not significant $\left[F(1,23)=1.79, M S_{\mathrm{e}}=5.97\right]$.

\section{EXPERIMENTS 3 AND 4}

The next two experiments involved two additional attempts at experimental realizations of enhanced visual distinctiveness. In the third study, each of the nine serial positions in experimental lists was associated with presentation of the digit in the position with a different color $^{2}$ 


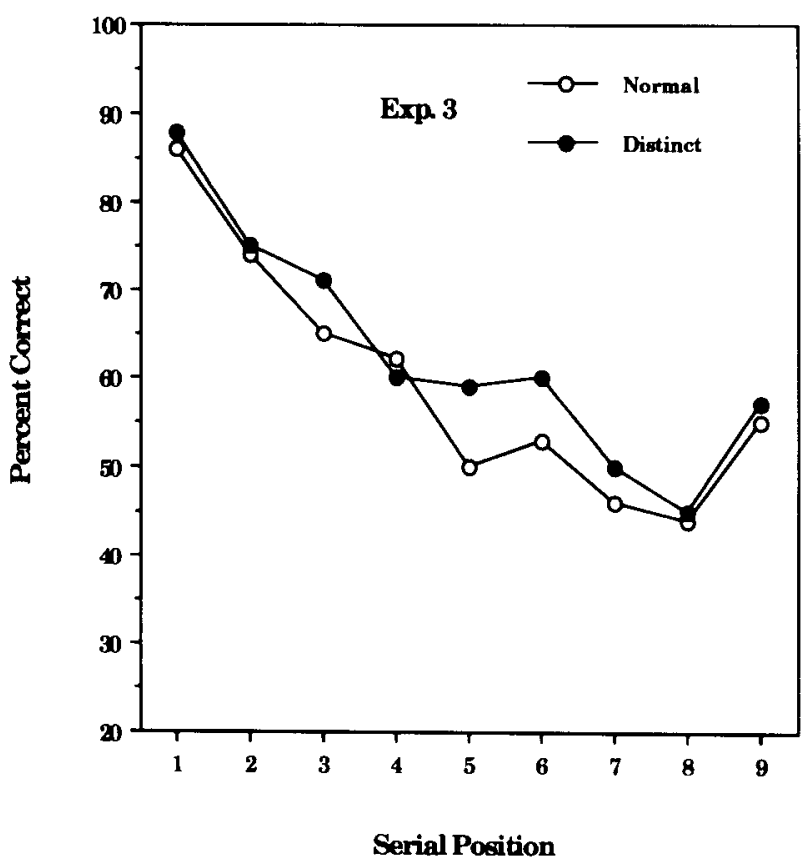

Figure 3. Percent correct ordered recall by serial position for normal- and changing-colors conditions (Experiment $\mathbf{3}$ ).

against a black background. These color-position combinations remained the same across all 10 lists, although they varied at random over subjects. Color and serial position were confounded in Experiment 3. Experiment 4 confounded color and digit: Each digit appeared in the same color across all 10 lists per subject, but in randomly selected serial positions. As in Experiment 3, the specific digit-color combinations varied across subjects.

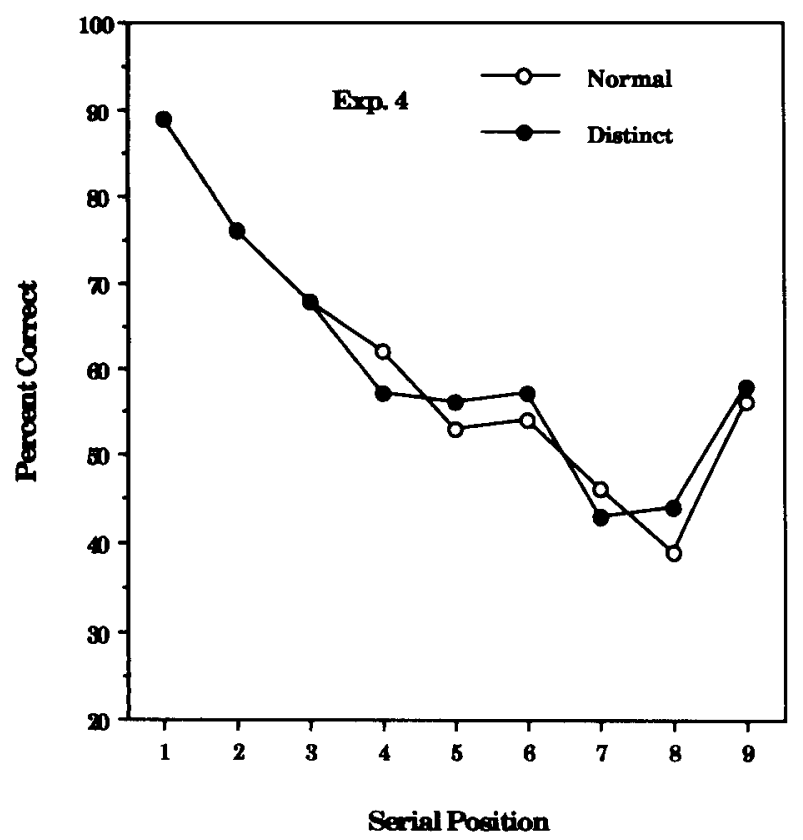

Figure 4. Percent correct ordered recall by serial position for normal- and changing-colors conditions (Experiment 4).

\section{Method}

The basic details of procedure were identical to those of Experiments 1 and 2 , including the within-subjects variation of normal and distinctive lists. In Experiments 3 and 4, subjects were also instructed that they did not have to learn or retain the specific color information.

There were 40 college-student subjects in Experiments 3 and 4, 22 subjects in Experiment 3, and 18 subjects in Experiment 4, randomly assigned to the two treatment orders.

\section{Results}

The results are presented in Figure 3 (Experiment 3) and Figure 4 (Experiment 4 ) in the form of serial-position curves. In Experiment 3, there was a marginally significant main effect of treatment $\left[F(1,20)=3.65, M S_{\mathrm{e}}=\right.$ $3.30]$, reflecting overall greater recall in the distinct condition. However, treatment $\times$ serial position effect was not significant $(F<1)$, and no advantage for the distinct condition was observed at the last serial positions.

The results for Experiment 4 are equally clear: No effect of variation of color was observed at any serial position. Here, there was neither a main effect of treatments nor any treatment $\times$ serial position interaction (both $F \mathrm{~s}<1$ ).

\section{EXPERIMENT 5}

Convinced at this point that further attempts with colorchange procedures were not going to be effective, we selected another dimension along which visually presented material can easily vary: spatial location. The experiment was identical in intent to one reported by Hitch and Morton (1975), who compared recall of digits presented in a single, fixed spatial location with recall of digits presented at different positions along a horizontal display. They failed to find any difference in recall. The present study was a replication of Hitch and Morton's study, with a potentially stronger spatial discriminability operation.

\section{Method}

Again, the basic procedure was the same as in Experiments 1-4. In control lists, digits always occurred in the center of the screen, in a fixed location. In experimental lists, digits appeared in nine locations arranged in a $3 \times 3$ layout, the center of the screen being the second element of the second row. Digits were assigned at random to locations across lists and subjects. Subjects were required to recall digits in temporal order of presentation, as usual; there was no requirement to learn or recall spatial location.

There were 18 college-student subjects in Experiment 5.

\section{Results}

Figure 5 shows the serial position curves of recall of control and varied spatial-location lists. The overall outcome is by now familiar: No reliable differences in recall were observed at any serial position. The $F$ ratios for the treatment main effect and treatment $\times$ serial-position interaction were both less than 1.0. These results are in complete agreement with Hitch and Morton's results, even though the present study added additional spatial cues (vertical cues, as well as just the horizontal differentiation used by Hitch and Morton). 


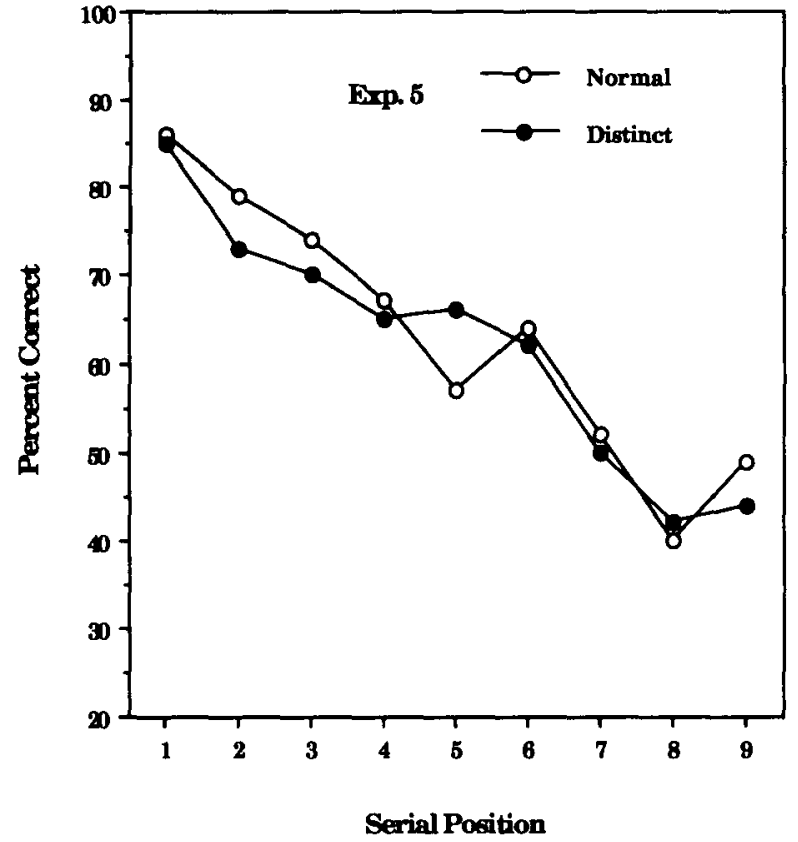

Figure 5. Percent correct ordered recall by serial position for normal- and distinct-location conditions (Experiment 5).

\section{EXPERIMENTS 6 AND 7}

The final two experiments examined visual-distinctiveness effects on recall in a manner quite different from the itemenhancement methods of Experiments 1-5. The experiments were prompted by the observation that in most memory experiments with visual presentation of material, no attempt is made to reduce or eliminate simultaneous and intervening visual stimulation from other sourcesfrom the display equipment itself, the response keyboard, or the testing environment in general. By way of contrast, auditory presentation of material most often occurs under conditions of low background noise and low levels of ambient auditory stimulation.

The intent of the experiment, then, was to compare the extent of recency effects in recall of visually presented material under the usual, visually noisy conditions with recall under conditions of minimized visual stimulation apart from presentation of the to-be-remembered material.

\section{Method}

The overall structure of the two experiments paralleled that of Experiments 1-5: Subjects recalled lists presented under both control and experimental conditions in a counterbalanced order. The control conditions in each study involved visual presentation of word (not digit) lists under "normal" viewing conditions, the word lists being presented on a CRT display in a normal, fully lighted laboratory room. The experimental conditions consisted of the same CRT presentation of to-be-remembered materials, but in a completely darkened room. The conditions were such that the dark-adapted subject could at most make out a faint outline of the CRT unit.

In Experiment 6, subjects were tested for immediate serial recall of the 10 8-word lists. In Experiment 7, the task was immediate free recall of 12-word lists. In all cases, because of the darkenedroom requirement, subjects spoke rather than wrote their responses.
The spoken responses were transmitted by microphone to the experimenter, who monitored and scored recall in an adjacent room. In both experiments, a microcomputer program created sets of word lists for each subject individually by random sampling without replacement from a pool of 200 common nouns. Lists were presented at a rate of one word per second in both studies, with $60 \mathrm{sec}$ allowed for recall.

There were 30 subjects in Experiment 6 and 20 subjects in Experiment 7 .

\section{Results}

Figures 6 and 7 show recall levels as a function of serial position and room condition for the serial- and free-recall studies, respectively.

Ordered recall. A 2 (condition: light room vs. dark room) $\times 8$ (serial position) analysis of variance was carried out on the ordered-recall data of Experiment 6 . The main effect of condition was highly significant $[F(1,29)=$ $\left.8.22, M S_{\mathrm{e}}=1.46, p<.01\right]$, indicating that recall was better in light-room conditions than in dark-room conditions. The effect of serial position was also significant $\left[F(7,203)=15.61, M S_{\mathrm{e}}=4.06, p<.001\right]$. The two factors did not interact $(F<1.0)$.

Free recall. A 2 (condition: light room vs. dark room) $\times 12$ (serial position) analysis of variance was carried out on the free-recall data of Experiment 7. The main effect of condition was marginally significant $[F(1,23)=$ 3.19, $M S_{\mathrm{e}}=1.25, p<.09$ ], indicating that dark-room performance was slightly better than light-room performance. The effect of serial position was highly significant $\left[F(11,253)=28.51, M S_{\mathrm{e}}=3.62, p<.001\right]$. There was no trace of an interaction of condition $\times$ serial position $(F<1.0)$.

Related studies. The conclusion that eliminating or at least minimizing extraneous visual stimulation does not

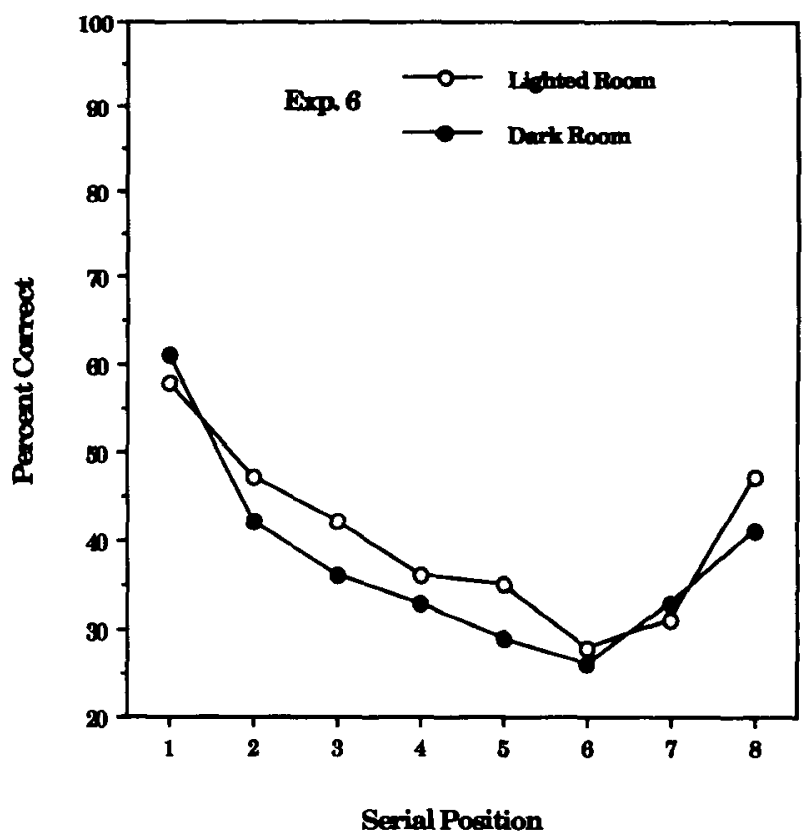

Figure 6. Percent correct ordered recall by serial pasition for lightroom and dark-room conditions (Experiment 6). 


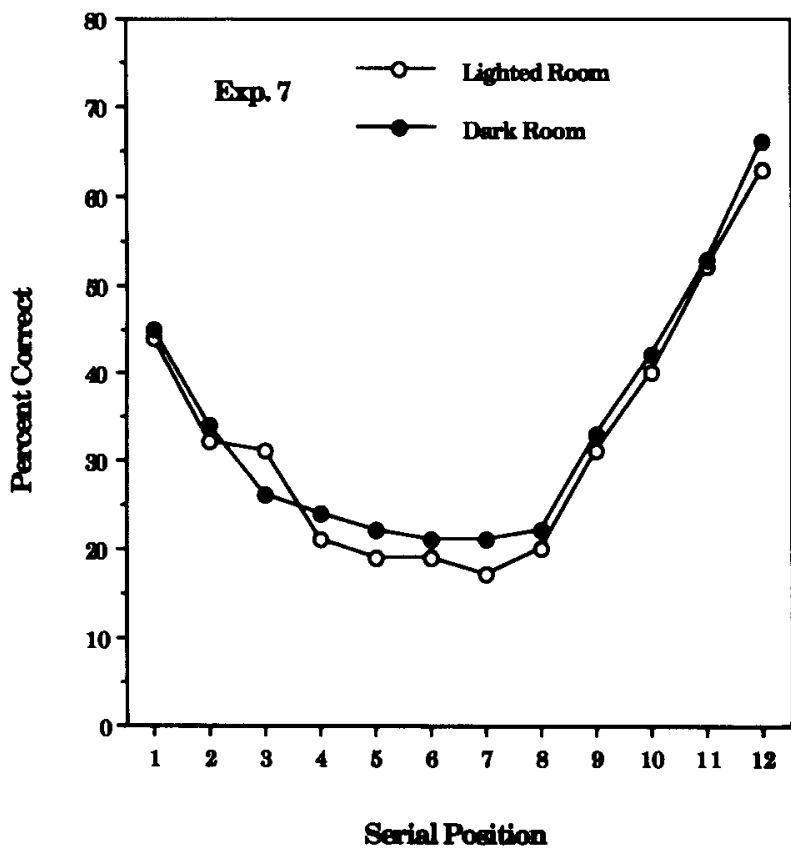

Figure 7. Percent correct free recall by serial position for lightroom and dark-room conditions (Experiment 7).

improve recall receives additional support from the results of two other studies we came across following the completion of these experiments. Hitch (1975) compared digitlist recall under light- and dark-room conditions. His rationale is worth quoting (p. 502): "With auditory presentation, great care is taken to minimize background noise but visual presentation typically occurs in a lighted room providing a large number of irrelevant stimuli. The use of written recall may exacerbate the situation." In a between-groups comparison of recall in lighted and darkened rooms, Hitch found, as we did in Experiment 6, that recall was in fact impaired by the dark-room treatment.

A more recent study relevant to the present outcomes was reported by Glenberg, Eberhardt, and Belden (1987), who had subjects view list items on a CRT through a viewpiece that excluded most extraneous visual stimulation during recall and throughout a retention interval. While these authors were concerned with the long-term modality effect rather than recency effects in immediate recall, they found that auditory recency still exceeded visual recency obtained under restricted viewing conditions.

\section{GENERAL DISCUSSION}

The experiments reported here started with the simple procedure of changing the last digit in a list to a new color (Experiment 1), then progressed through procedure variations introducing more extensive changes in color and color differences of presented material in Experiments 2-4. Experiment 5 represented an attempt to increase visual distinctiveness and recall performance through variation of spatial location of presentation, in line with suggestions that spatial encoding is characteristic of the processing of visual stimuli (Nairne, 1988; Penney, 1989). In none of the studies were we able to obtain any effects of the distinctive operations. In particular, there was no enhanced recall of the final list item in any of the studies.

It may be helpful at this point to compare this set of results with the serial-position effects typically generated by auditory as compared with visual sequences. ${ }^{3}$ We tested 23 additional subjects with visual presentation of lists, following essentially the same procedures used in Experiments 1-5 for "normal" conditions, and also with auditory presentation with tape-recorded sequences. In both cases the response mode was the same, with subjects typing single-digit responses on a computer keyboard. Modality of presentation was blocked with order of blocks and list-to-modality assignments counterbalanced across subjects. There were 10 lists per block.

Figure 8 shows the serial-position curves for auditory and visual presentation. The results are entirely typical; we found a much larger recency effect for auditory presentation with small or no differences elsewhere. Indeed, the elusive condition $\times$ serial position interaction was here observed to be significant $[F(8,176)=3.82, p<.001]$. The observed difference between auditory and visual presentation for the final list item is very much larger than any such difference between distinctive and normal presentations in Experiments 1-5.

To relate the present results as a whole to current theoretical understanding of modality and recency effects, it is helpful to view them from the position outlined by Nairne (1988). A central contention of Nairne's position is that modality differences in recency effects can be understood without any appeal to a "special" status or characteristic of auditory stimuli, such as an inherently

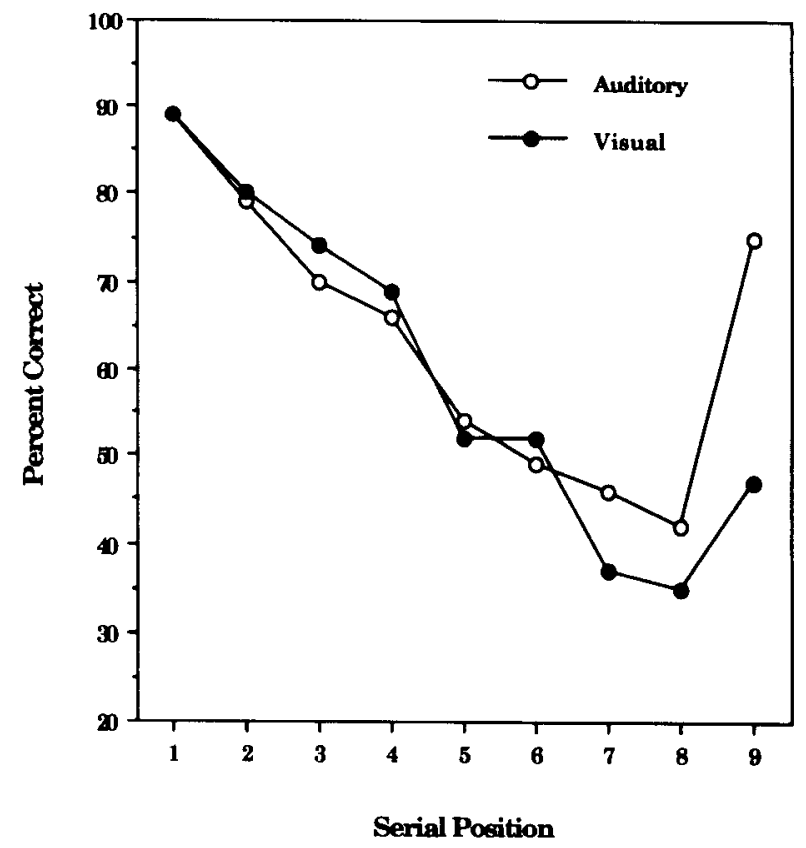

Figure 8. Percent correct ordered recall by serial position for auditorily and visually presented items. 
greater temporal discriminability of an auditory as opposed to a visual sequence. Instead, Nairne argues, auditory recency typically exceeds visual recency for other reasons. Among these are the typically greater amount of visual interference with visual presentation of material, as well as the tendency of subjects to encode visually presented verbal material into a speech-linguistic code, omitting potentially useful visual features.

The first of these contentions is clearly disconfirmed by the results of Experiments 5 and 6: Reducing extraneous visual stimulation to a near-zero level does not produce any increased recency. As Nairne (1988) acknowledges, the evidence for increased recency with decreased visual interference is "mixed," citing Hitch (1975) as a negative outcome and Glenberg et al. (1987) as a positive outcome. If, however, consideration is limited to immediate recall under more or less standard conditions (Hitch's experiment, and the current Experiments 5 and 6), then the evidence to date is that there is no recency enhancement attendant on reduced visual interference.

The present experiments do not directly address the second of Nairne's (1988) suggestions, that recoding of visually presented materials works against use of visual features to organize or control recall. However, on the surface at least, this view seems to predict that the addition of perceptually discriminating nonverbal information such as color and spatial location would have some positive influence on recall. This prediction was not borne out by the present results.

In support of the recoding argument, Nairne (1988) also cites studies that employed nonverbal or hard-to-recode visual stimuli, noting that they can produce appreciable recency effects (e.g., Broadbent \& Broadbent, 1981). A recent study by Battacchi, Pelamatti, and Umilta (1990) seems, in fact, to have produced strikingly large recency effects for visual sequences presented in a vertical, spatially distributed array with materials (acoustically confusable syllables) that might encourage visual-spatial encoding. The possibility thus remains that methods other than the ones we employed can, in fact, augment recency effects for visually presented materials.

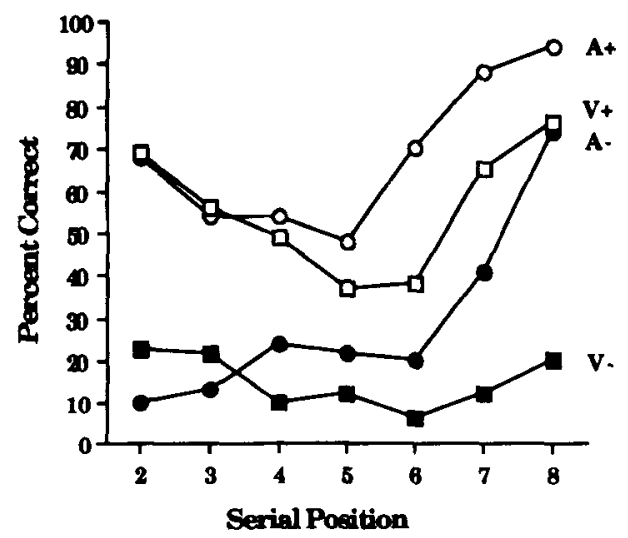

We want to conclude by discussing an important but seldom considered characteristic of the recency effect obtained with auditory presentation, one that bears directly on the issue of commonality of processes. This is the nearindependence of recall of the last item in a list when conditionalized on recall or nonrecall of the preceding item. This effect was first reported some time ago (Morton, 1970; Murdock, 1968). Figure 9 presents two further examples of this. The left panel of Figure 9 shows recall probabilities conditionalized on recall and nonrecall of the preceding item for auditory and visually presented word lists in an immediate-serial-recall task (data from Madigan, 1971). The main point of these data, for present purposes, is the near-independence of recall of the last auditory item and the strong dependence of recall of the last item for visual presentation. The right panel of Figure 9 shows the same analysis for the auditory-visual data presented in Figure 8, and it makes precisely the same point. By way of contrast, consider the conditionalized data pooled over Experiments 1-4 of the present series (Figure 10); they contain no hint of an independence effect for visually distinctive materials.

It would be of considerable interest at this point to determine if the independence-dependence pattern of outcomes occurs under those experimental conditions that have complicated things so much for PAS theory, such a mouthing, lip-reading, delay of recall, and long-term modality effects. The long-term modality effect, for example, could not be claimed to represent the same basic processes as the modality effect in immediate recall if its patterns of dependence-independence do not resemble the kinds of outcomes presented here. More directly relevant to the present studies, the same could be said for demonstrations of enhanced recency for visual materials, such as the results of Battacchi et al. (1990), or the enhanced recency with changing-state visual stimuli reported by Kallman and Cameron (1989).

In closing, we note first that the independence issue is by no means settled, as Glenberg (1990) has recently reported correlational analyses of auditory and changingstate visual stimuli in which he finds positive correlations

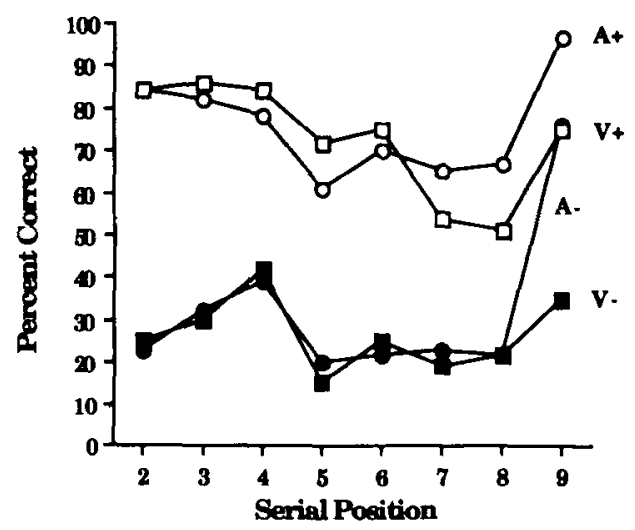

Figure 9. Recall conditionalized on correct $(+)$ or incorrect $(-)$ recall of the preceding item. Left panelData from Madigan (1971). Right panel-Data from the auditory-visual study. 


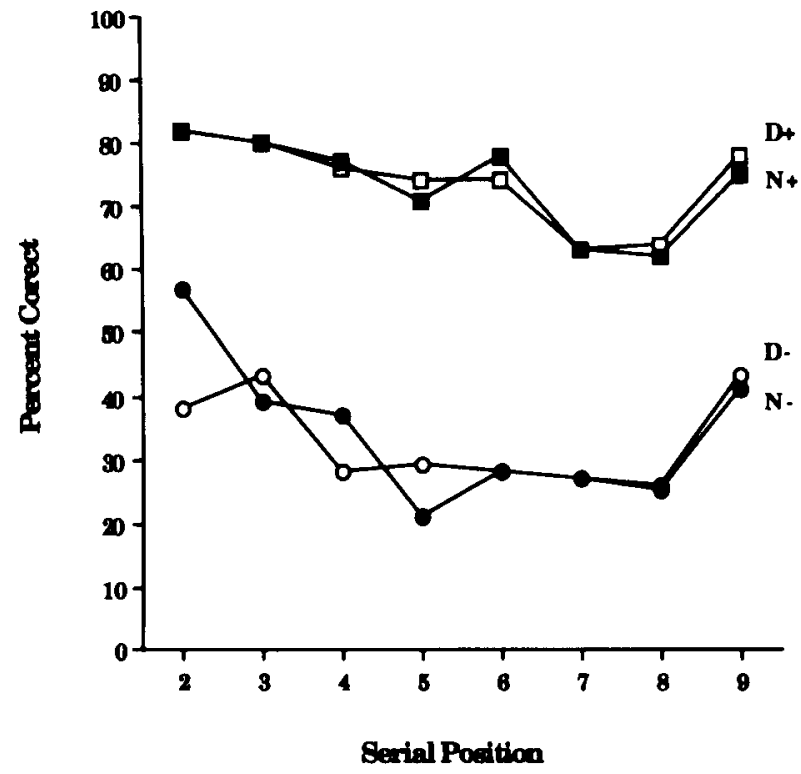

Figure 10. Conditionalized recall for distinctive (D) and normal (N) conditions, with data pooled over Experiments 1-4.

between derived "recency scores" for these stimuli. Pending resolution of differences in definition and measurement of independence, we would like to suggest an interpretation of the auditory-independence effect as it has shown up in the conditional probability analyses: The effect seems entirely consistent with the PAS-like view that the modality effect in immediate recall represents a contribution of a sensory and not a cognitive system. Stretching things perhaps a bit, the finding that recall of the last item of an auditory sequence is uncorrelated with recall of preceding items is like Galton's finding that tests of basic sensory functions are poorly correlated with measures of intellectual functioning (see Jensen, 1980, pp. 137-140). On a less grand scale, consider Murdock's (1968) analysis of auditory digit-span recall with separate position curves for the best and worst subjects, defined in terms of overall performance level: These curves are very different at all serial positions except for the last.

\section{REFERENCES}

Battacchi, M. W., Pelamatti, G. M., \& Umiltä, C. (1990). Is there a modality effect? Evidence for visual recency and suffix effects. Memory \& Cognition, 18 , 651-658.

Broadbent, D. E., Broadbent, M. H. P. (1981). Recency effects in visual memory. Quarterly Joumal of Experimental Psychology, 33A, $1-15$.

Crowder, R. G. (1986). Auditory and temporal factors in the modality effect. Journal of Experimental Psychology, 12, 268-278.
Crowder, R. G., a Morton, J. (1969). Precategorical acoustic storage (PAS). Perception \& Psychophysics, 5, 365-373.

GLENBERG, A. M. (1984). A retrieval account of the long-term modality account. Joumal of Experimental Psychology: Learning, Memory. \& Cognition, 9, 231-255

GLENBERG, A. M. (1987). Temporal context and recency. In D. S. Gorfein \& R. R. Hoffman (Eds.), Memory and learning: The Ebbinghaus Centennial Conference (pp. 173-191). Hillsdale, NJ: Erlbaum.

GLENBERG, A. M. (1990). Common processes underlie enhanced recency effects for auditory and changing-state stimuli. Memory \& Cognition, 18, 638-650

Glenberg, A. M., Eberhardt, K. A., \& Belden, T. M. (1987). The role of visual interference in producing the long-term modality effect. Memory \& Cognition, 15, 504-510.

Glenberg, A. M., SWanson, N. G. (1986). A temporal distinctiveness theory of recency and modality effects. Journal of Experimental Psychology: Learming, Memory, \& Cognition, 12, 3-15.

Hitch, G. J. (1975). The role of attention in visual and auditory suffix effects. Memory \& Cognition, 3, 501-505.

HITCH, G., MorTon, J. (1975). The unimportance of explicit spatial information in serial recall of visually presented lists. Quarterly Joumal of Experimental Psychology, 27, 161-164.

JenSEN, A. R. (1980). Bias in mental testing. New York: The Free Press.

Kallman, H. J., Cameron, P. (1989). Enhanced recency effects with changing-state and primary-linguistic stimuli. Memory \& Cognition, 17, 318-328.

MadigAN, S. A. (1971). Modality and recall order interactions in shortterm memory for serial order. Journal of Experimental Psychology, 87, 294-296

Morton, J. N. (1970). A functional model for memory. In D. Norman (Ed.), Models of human memory (pp. 203-254). New York: Academic Press.

MURDOCK, B. B., JR. (1968). Serial order effects in short-term memory. Journal of Experimental Psychology Monographs, 76(Suppl., Pt. 2), $1-15$.

NaIRNE, J. S. (1988). A framework for interpreting recency effects in immediate serial recall. Memory \& Cognition, 16, 343-352.

PENney, C. G. (1989). Modality effects and the structure of short-term verbal memory. Memory \& Cognition, 17, 398-422.

\section{NOTES}

1. One suggestion that has been made concerning our failure to find an effect of visual distinctiveness is that subjects received no practice in the distinct conditions and may have required a few trials to be able to take advantage of the visual distinctiveness. Any initial negative effects of the distinct manipulations would wash out any advantage that may have existed on later trials. To test this hypothesis, we divided the data from the first five experiments for which individual trial data were available into two blocks of five trials each, representing the first and second half-trials. A treatment $\times$ block $\times$ serial-position analysis of variance was then performed. In only one case (Experiment 4) did the critical treatment $\times$ block $\times$ serial-position interaction reach significance, but the pattern of data in that study indicated that recency in the distinct condition was actually greater in the first block than in the second block.

2. The colors were BASICA medium-resolution graphics 1-9.

3. The inclusion of these data was suggested by a reviewer

(Manuscript received November 6, 1989; revision accepted for publication December 21, 1990.) 